\title{
Electronic structure and magnetic properties of $\mathrm{Li}_{2} \mathrm{ZrCuO}_{4}$ - a spin 1/2 Heisenberg system in vicinity to a quantum critical point
}

\author{
M. Schmitt, ${ }^{1}$ J. Málek, ${ }^{2,3}$ S.-L. Drechsler, ${ }^{3}$ and H. Rosner ${ }^{1}$ \\ ${ }^{1}$ Max-Planck-Institut für Chemische Physik fester Stoffe, Nöthnitzer Str. 40, D-01187 Dresden, Germany \\ ${ }^{2}$ Institute of Physics, ASCR, Prague, Czech Republic \\ ${ }^{3}$ Leibniz-Institut für Festkörper- und Werkstoffforschung Dresden, P.O. Box 270116, D-01171 Dresden, Germany
}

\begin{abstract}
Based on density functional calculations, we present a detailed theoretical study of the electronic structure and the magnetic properties of the quasi-one dimensional chain cuprate $\mathrm{Li}_{2} \mathrm{ZrCuO}_{4}$ $\left(\mathrm{Li}_{2} \mathrm{CuZrO}_{4}\right)$. For the relevant ratio of the next-nearest neighbor exchange $J_{2}$ to the nearest neighbor exchange $J_{1}$ we find $\alpha=-J_{2} / J_{1}=0.22 \pm 0.02$ which is very close to the critical point at $1 / 4$. Owing this vicinity to a ferromagnetic-helical critical point, we study in detail the influence of structural peculiarities such as the reported $\mathrm{Li}$ disorder and the non-planar chain geometry on the magnetic interactions combining the results of LDA based tight-binding models with LDA $+U$ derived exchange parameters. Our investigation is complemented by an exact diagonalization study of a multi-band Hubbard model for finite clusters predicting a strong temperature dependence of the optical conductivity for $\mathrm{Li}_{2} \mathrm{ZrCuO}_{4}$.
\end{abstract}

PACS numbers:

\section{INTRODUCTION}

Low dimensional magnetism has always attracted great interest in solid state physics and chemistry. Especially, the phase diagram of low dimensional spin-1/2 lattices has been investigated extensively, both theoretically and experimentally. For low dimensions and spin-1/2, the influence of quantum fluctuations becomes crucial for the ground state of the system. The role of quantum fluctuations is even more pronounced if the system under consideration exhibits strongly frustrated interactions.

Taking only nearest neighbor interactions into account, pure geometrical frustration in two dimensions (2D) can be realized by special symmetries, i.e triangular or Kagomé lattices. Prominent real material realizations for such lattices are $\kappa$-(BEDT-TTF $)_{2} \mathrm{X}$ (Ref 1 ) and $\mathrm{ZnCu}_{3}(\mathrm{OH})_{6} \mathrm{Cl}_{2} \cdot 2$ Another possibility to realize frustrated couplings are competing nearest neighbor (NN) and next nearest neighbor (NNN) interactions. This way, the unfrustrated NN Heisenberg square lattice becomes frustrated by adding antiferromagnetic (AFM) interactions to NNN. The ground state of this model is determined by the ratio $\alpha=-J_{2} / J_{1}$ between NNN and NN exchange interaction. Including a tiny, but non-vanishing coupling between the magnetic planes that is always present in real materials, for small $\alpha$ an antiferromagnetic ground state is observed like in all undoped HTSC parent compounds $\stackrel{3}{=}$ For large $\alpha$ the so called columnar order is expected, as found in $\mathrm{Li}_{2} \mathrm{VSiO}_{4}$ or $\mathrm{Li}_{2} \mathrm{VGeO}_{4}, 4,5,6,7,8 \mathrm{Be}-$ tween these commensurably ordered phases, theory predicts a spin liquid ground state. The possible realization of this spin liquid ground state in $\mathrm{PbVO}_{3}$ is presently under debate. 9.10

In one dimension (1D), pure geometrical frustration due to NN exchange is impossible, but frustration by competing NN and NNN exchanges may occur in close analogy to the $2 \mathrm{D}$ square $J_{1}-J_{2}$ model. The phase diagram of this seemingly simple model is very rich. De- pending on the frustration ratio $\alpha$, a variety of ground states was observed in corresponding quasi-1D systems: (i) ferromagnetically ordered chains in $\mathrm{Li}_{2} \mathrm{CuO}_{2}{ }^{11}(0 \leq$ $\alpha \leq 0.25$, within an effective single-chain analysis ignoring the antiferromagnetic inter-chain interaction ${ }^{12}$ ), (ii) helical order with different pitch angles in $\mathrm{LiVCuO}_{4}$, $\mathrm{LiCu}_{2} \mathrm{O}_{2}$, and $\mathrm{NaCu}_{2} \mathrm{O}_{2} 13,14,15,16,17,18(\alpha>0.25)$ or spin gap behavior like in the famous spin-Peierls compound $\mathrm{CuGeO}_{3}{ }^{19}(-\alpha>0.241)$, i.e. both exchange couplings are antiferromagnetic.

Very recently, large interest focused on chain systems that are close to the quantum critical point (QCP) at $\alpha=0.25$. In the vicinity of a QCP, the system is expected to answer in a pronounced way to small external perturbations like magnetic fields or pressure. In addition, even very small additional exchange paths, especially the inter-chain coupling, become important for the magnetic properties of the system. A most promising compound to study the close vicinity to the QCP is $\mathrm{Li}_{2} \mathrm{ZrCuO}_{4}$, where a frustration ratio of $\alpha=0.29$ was reported from the evaluation of susceptibility and specific heat data. 20 The expected strong field dependence of the specific heat from magnetic fields up to 9 Tesla was observed, although the NN exchange $J_{1}$ was estimated to be rather large in the order of $300 \mathrm{~K}$. The vicinity of $\mathrm{Li}_{2} \mathrm{ZrCuO}_{4}$ to the QCP was supported by preliminary band structure calculations that obtained a frustration ratio $\alpha \sim 0.23$.

In this paper, we report a detailed electronic structure study based on density functional calculations. In particular, we investigate the influence of the experimentally observed $\mathrm{Li}$ disorder ${ }^{21}$ for one of the crystallographic $\mathrm{Li}$ sites on the magnetic properties. We discuss the dependency of the exchange integrals and the frustration ratio $\alpha$ on the strong Coulomb repulsion within the $\mathrm{Cu}$ $3 d$ orbitals. In addition, we elucidate the crucial importance of the structural distortion of the $\mathrm{CuO}_{2}$ chains in $\mathrm{Li}_{2} \mathrm{ZrCuO}_{4}$. This distortion is responsible for a reduction of both the inter-chain coupling and the NNN exchange 
$J_{2}$, as a consequence placing the system very close to the $\mathrm{QCP}$.

\section{METHODS}

For the electronic structure calculation a fullpotential non-orthogonal local-orbital minimum-basis scheme (FPLO5.00-19 and FPLO7.00-28) $\stackrel{22,23}{ }$ within the local (spin) density approximation (L(S)DA) was used. In the scalar relativistic calculations the exchange and correlation potential of Perdew and Wang was used. ${ }^{24}$ We also applied the general gradient approximation (PerdewBurke-Ernzerhof ${ }^{25}$ ) for the exchange and correlation potential to check whether this influences the LDA results. Neither for the band structure nor for the total energy differences we found any significant changes. For the basis the following states were taken into account: $\mathrm{Cu}(3 s 3 p) / 4 s 4 p 3 d, \mathrm{Li} 1 s /(2 s 2 p)+3 d, \mathrm{O}(2 s 2 p 3 d), \mathrm{Zr}$ $(4 s 4 p) /(5 s 5 p 4 d)$ (notation: semi-core states / valence states). The $\mathrm{Cu} 3 s 3 p, \mathrm{Li} 1 s$ and $\mathrm{Zr} 4 s 4 p$ states were treated as semi-core states due to the large extension of their wave functions. The unoccupied states Li $2 p 3 d$, O $3 d$ and Zr $5 p$ were considered as hybridization states to complete the basis. All lower laying states were treated as core states. To ensure accuracy of the total energy $300 \mathrm{k}$-points in the irreducible part of the Brillouin zone were used.

To model the $\mathrm{Li}(1)$ split position of the system (see below), we employed the virtual crystal approximation (VCA) and coherent potential approximation (CPA). Alternatively super-cells with different $\mathrm{Li}(1)$ orders have been studied. To treat the strong on-site Coulomb repulsion for $\mathrm{Cu} 3 d$ orbitals explicitly, the $\mathrm{L}(\mathrm{S}) \mathrm{DA}+U$ method 26 with $U_{3 d}$ in the representative range from $5.5 \mathrm{eV}$ to $8.0 \mathrm{eV}$ and $I=1 \mathrm{eV}$ for the intra atomic exchange have been used.

To study some aspects of the magnetic properties of the system a tight binding model (TBM) was derived from the LDA-calculations to find the transfer integrals for a single-band effective extended Hubbard model which subsequently has been mapped onto a corresponding 3D Heisenberg model.

In addition, exact diagonalization studies of a fiveband $p d$ extended Hubbard model and its mapping onto the 1D-Heisenberg model using finite open $\mathrm{Cu}_{n} \mathrm{O}_{2 n+2}$ clusters have been carried out.

\section{STRUCTURE}

The $\gamma$-phase of $\mathrm{Li}_{2} \mathrm{ZrCuO}_{4}{ }^{21,27}$ crystallizes in an orthorhombic space group and is shown in Fig. 17a. The compound contains distorted $\mathrm{CuO}_{4}$ plaquettes which are arranged as edge-shared $\mathrm{CuO}_{2}$ chains with an $\mathrm{Cu}-\mathrm{O}-\mathrm{Cu}$ bond angle of $94.13^{\circ}$. These edge shared $\mathrm{CuO}_{2}$ chains form layers together with the $\operatorname{Li}(2)$ atoms in the $y z$-plane

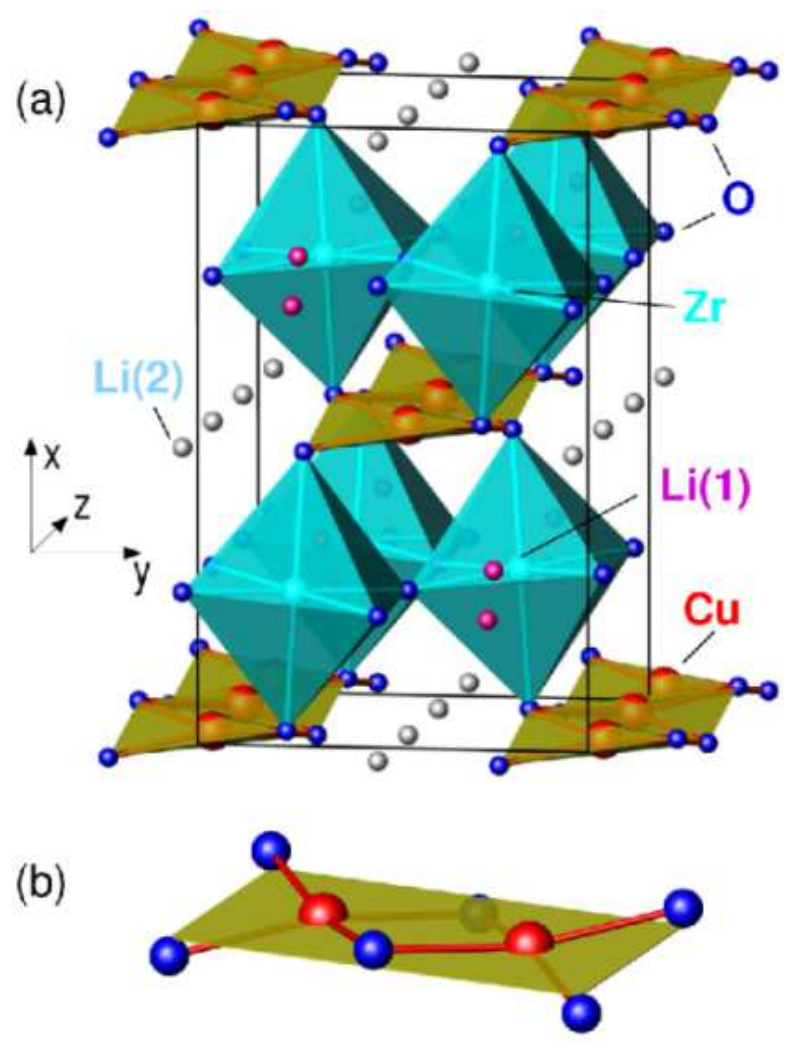

FIG. 1: (Color online) (a) Crystal structure of $\mathrm{Li}_{2} \mathrm{ZrCuO}_{4}$ : Chains of edge-shared $\mathrm{CuO}_{4}$ plaquettes (yellow) run along the $z$-direction and are connected by $\mathrm{ZrO}_{6}$-octraheda (light blue). The fully occupied $\operatorname{Li}(2)$ site (gray) lies in the chain plane. The $\mathrm{Li}(1)$ split position (pink) alternates with the $\mathrm{ZrO}_{6}$ octahedra. (b) Section of the $\mathrm{Cu}-\mathrm{O}$ chain: The $\mathrm{O}$ atoms of a plaquette are deflected from the mirror plane (shown in yellow).

interconnected by $\mathrm{ZrO}_{6}$ octrahedra. The alternating arrangement of the connecting $\mathrm{ZrO}_{6}$ octrahedra lead to the deviation of the $\mathrm{CuO}_{2}$ chains from the ideal planar geometry. In Fig. 10 the deflection of the $\mathrm{O}$ atoms from the chain plane is illustrated in detail.

As the $\mathrm{CuO}_{4}$ plaquettes exhibit a localized, effective spin $1 / 2$, their linking and surrounding is of main importance for the formation of the magnetic ground state. A first structural analysis suggests sizable ferromagnetic NN exchanges caused by the close to $90^{\circ} \mathrm{Cu}-\mathrm{O}-\mathrm{Cu}$ bond angle according to the Goodenough-Kanamori-Anderson rule, provided the direct $\mathrm{Cu}-\mathrm{Cu}$ transfer integral can be ignored. 28

A further structural characteristic of the $\gamma$-phase is a split position for $\mathrm{Li}(1)$ which is placed between the chain layers. The distance of the $\operatorname{Li}(1)$ atoms from the high-symmetry position 29 is $0.37 \AA$. While the influence of the split position of $\mathrm{Li}(1)$ (corresponding to $50 \%$ disorder within a classical picture, ignoring possible tunneling processes of $\mathrm{Li}(1)$ between the two sites of the split position) to the magnetic properties is investigated carefully, we neglect the small disorder of $3-5 \%$ at the $\operatorname{Li}(2)$ and 
$\mathrm{Cu}$ site in our theoretical calculations as a good approximation.

It is well known that in the vicinity of a QCP small changes in any parameter can be of crucial relevance for the ground state realized by the system. Therefore, the above mentioned structural peculiarities of $\mathrm{Li}_{2} \mathrm{ZrCuO}_{6}$ raise the following two questions especially related to their influence on the electronic and magnetic properties. (i)How does the modeling of the $\mathrm{Li}(1)$ split position influences the calculated ground state? As there exists no standard procedure in band structure codes to treat split positions we suggest various classical (i.e. within the adiabatic approximation) approaches. (ii) To which extend does the distortion of the $\mathrm{CuO}_{4}$ plaquettes influence the placement of the compound in the $J_{1}-J_{2}$ phase diagram? Therefore a fictitious structure with ideal planar chain geometry was also considered $\underline{30}$

\section{RESULTS AND DISCUSSION}

\section{A. Modeling the $\mathrm{Li}(1)$ split position}

The vicinity of $\mathrm{Li}_{2} \mathrm{ZrCuO}_{4}$ to a $\mathrm{QCP}$ requires a careful check of the structural description of the compound as a basis for our theoretical calculation. Therefore the treatment of the $\operatorname{Li}(1)$ split position might be crucial, although the different models may only exhibit subtle differences. In the following we suggest different models to handle the split position and discuss the results with respect to the influence of these model assumptions on the relevant states and related dispersions.

(i) To start from the most simplest approximation we performed electronic structure calculation for $\operatorname{Li}(1)$ placed at the high-symmetry position (see Fig. 2(SYM)).

(ii) As an alternative structural model we carried out VCA calculations (see Fig. 2 (VCA)). This approach occupies both sites of the split position simultaneously by a half of a $\mathrm{Li}$ atom. Each of this modified $\mathrm{Li}$ atoms is constructed by carrying the half number of valence electrons and a modified core to conserve charge. For the VCA calculations the distance between the two sites of the split position has to be enlarged to suppress the overlap of the $\mathrm{Li}$ valence states and ensure the convergence of the calculation. ${ }^{31}$ Also asymmetric charge distributions between the two sites of a split position (as e.g. 0.8 and $0.2 \mathrm{Li}$ ) lead to nearly identical dispersion of the relevant states as long as the total charge of one Li atom per split position is conserved. However, deviations of the total charge of a split position cause sizable changes in the electronic structure.

This is also the reason why the description of the $\operatorname{Li}(1)$ split position by CPA fails, caused by the loss of charge balance at the split position in the later approximation. In contrast to the VCA, where the total charge at each split position sums up to one $\mathrm{Li}$ atom, the random occupation of the $\mathrm{Li}(1)$ split sites in the CPA also generates cases with empty and double occupied $\operatorname{Li}(1)$ sites with
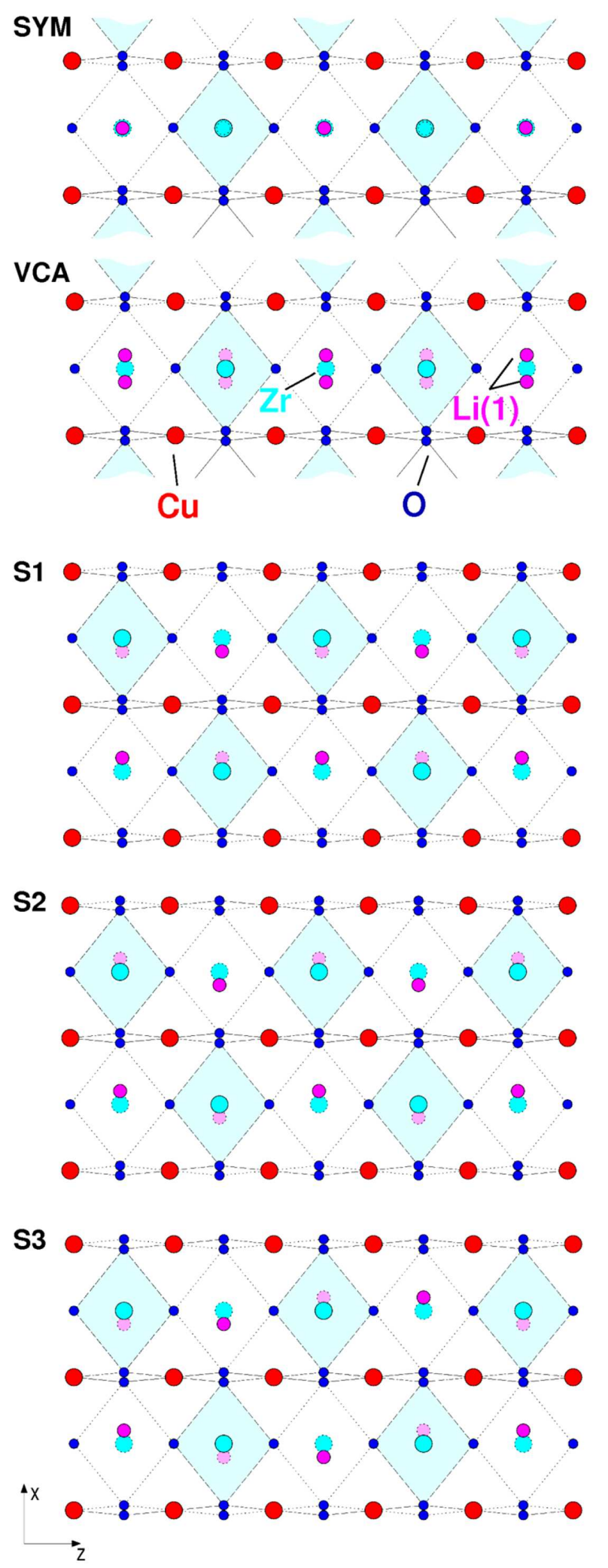

FIG. 2: (Color online) Different structural models to treat the Li(1) split position. From top to bottom: SYM - highsymmetry position for $\mathrm{Li}(1)$, VCA - both sites occupied by partial Li(1) atoms, S1 to S3 - super-cells with different Li(1) pattern. In $\mathrm{S} 1$ and $\mathrm{S} 2$ the $\mathrm{Li}(1)$ atoms along the chains are shifted parallel while in $\mathrm{S} 3$ the $\mathrm{Li}(1)$ atoms alter along the chain direction. $\mathrm{S} 1$ and $\mathrm{S} 2$ differ in the Li(1) inter-chain arrangement. The slight tilting of the $\mathrm{ZrO}_{6}$-octrahedra (see Fig. 1) is not depicted for the sake of simplicity (full lines foreground, dashed lines background). 


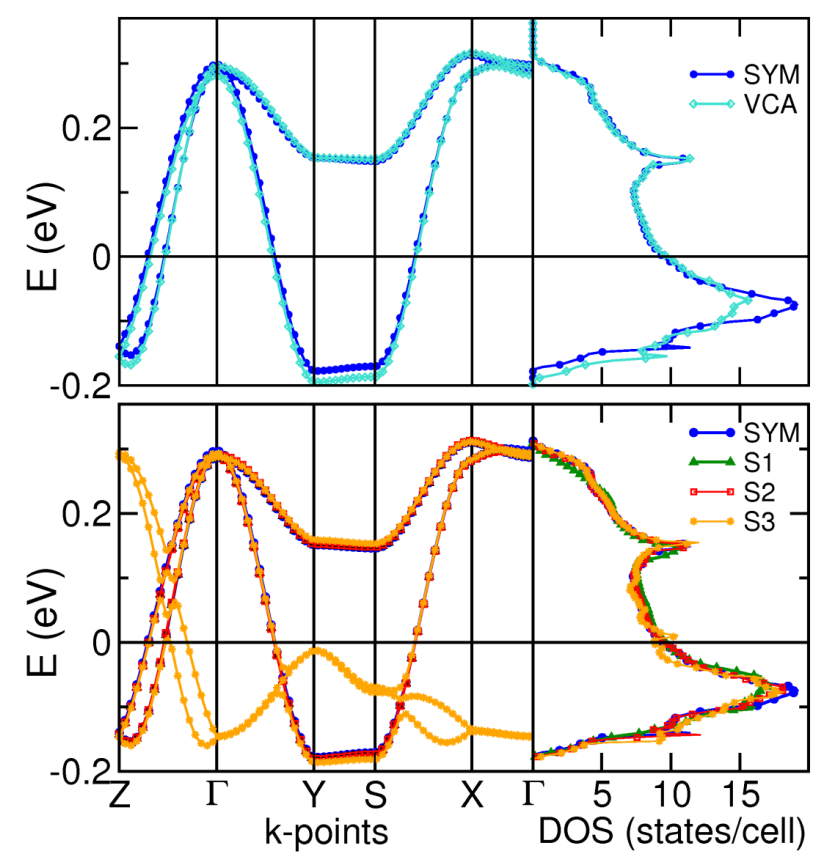

FIG. 3: (Color online) Calculated LDA density of states and band structure for the physical relevant states using different structural models for the Li split position. The comparison shows just minor differences.

dramatic influence on the band structure and the total energy.

(iii) As both latter approaches do not touch the local symmetry, within a further model super-cells with different $\operatorname{Li}(1)$ patterns have been calculated, breaking the local symmetry at the split position but suffer from long range order (see Fig. 2 S1-S3). In the two upper panels the super-cells were constructed by a reduction of the space group symmetry. The $\mathrm{Li}(1)$ atoms along the chains are all shifted in the same direction while the $\mathrm{Li}(1)$ atoms of neighboring chains are parallel (S1) or anti-parallel (S2) shifted. The third super-cell (Fig. 2 S3) shows an alternating displacement of the $\operatorname{Li}(1)$ atoms along the chain obtained by a doubling of the cell in $y$-direction.

The resulting bands and density of states for the different structural models show a very similar total behavior (see Fig. 31). Small deviations in VCA calculations can be observed in the bonding region of the valence band around -5.5 and $-2.0 \mathrm{eV}$ (not shown) in comparison to the high symmetry model. These differences can be understood by the influence of the two modified Li cores to the crystal potential and the corresponding shift of Li-O states.

Comparing the density of states and LDA band structure for the anti-bonding $d p \sigma^{*}$ states, which are relevant for the magnetic exchanges and therefore the magnetism of the system, we found only tiny differences. (i) The VCA model results in an slightly larger bandwidth compared to the other models. (ii) The high symmetry and super-cell models nearly fit perfect. The additional bands of the S3 super-cell with an alternating Li pattern can be understood due to the doubling of the primitive cell having its origin in the symmetry breaking of the Li pattern.

\section{B. Electronic Structure and Magnetic Properties}

As the different structural models for the Li(1) split position show no significant difference in behavior for the relevant low energy states (see Fig. 3), the electronic structure and the microscopic magnetic model are discussed for the SYM model as a good representative for all different structural models. To estimate more quantitatively the differences caused by the choice of the structural model the results for the SYM and the VCA models are compared.

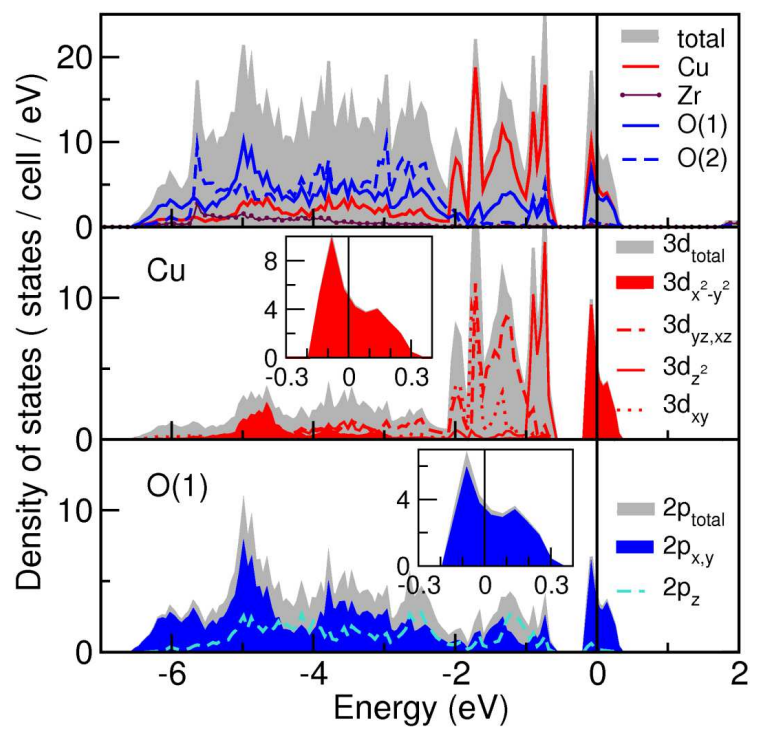

FIG. 4: (Color online) Calculated atom and orbital resolved density of states (LDA) for $\mathrm{Li}_{2} \mathrm{ZrCuO}_{4}$ applying the SYM model for the $\mathrm{Li}(1)$ split position (see text). The Li contribution to the valence band is negligible. $\mathrm{Cu}$ and $\mathrm{O}$ dominate the anti-bonding $d p \sigma^{*}$ band.

In Fig. 4 the atom and orbital resolved density of states for $\mathrm{Li}_{2} \mathrm{ZrCuO}_{4}$ are shown. The valence band has a width of about $7 \mathrm{eV}$, comparable with other chain cuprates and is dominated by $\mathrm{Cu}$ and $\mathrm{O}$ states, especially at the lower edge of the valence band around $-6 \mathrm{eV}$ (bonding states) and at the energy range close to zero (anti-bonding states). States of the $\mathrm{Zr}-\mathrm{O}$ octrahedra appear in the middle part of the valence band. Their contribution to the states close to the Fermi energy is negligible.

The metallic character of the LDA results is in contradiction to the experimentally observed insulating ground state. This effect is a well known shortcoming of the LDA as it underestimates the strong correlations in the $\mathrm{Cu}$ $3 d$ states. The related strong Coulomb repulsion on $\mathrm{Cu}$ sites can be considered explicitly by a model approach (e.g. a single-band Hubbard model using tight-binding 


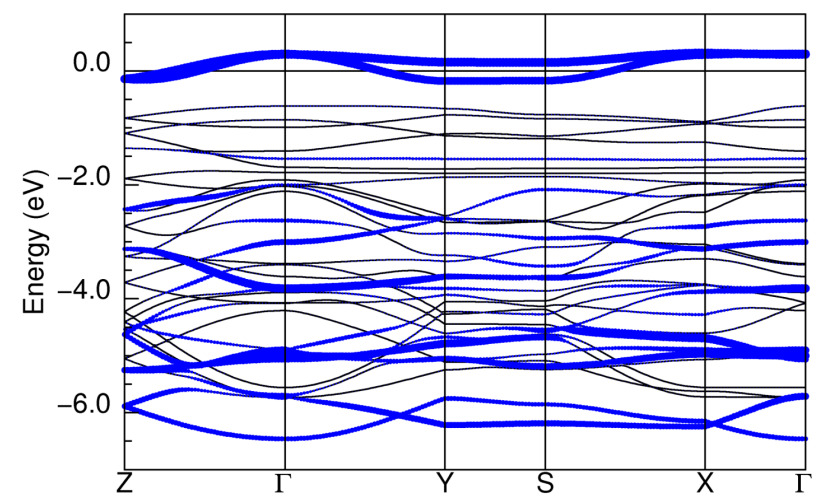

FIG. 5: (Color online) Valence band together with the band characters of the $\mathrm{Cu} 3 d$ and $\mathrm{O} 2 p$ anti-bonding states (blue) indicated by the line width. The band at Fermi energy is dominated by $\mathrm{Cu}$ and $\mathrm{O}$ anti-bonding $d p \sigma^{*}$ states.

parameters derived from the LDA) or by $\mathrm{LDA}+U$ calculations. Both methods were applied in this study and lead to an insulating ground state in agreement with the experimental data. Nevertheless, the LDA calculation delivers important insights into the electronic structure and provides the relevant bands for the development of a microscopically based picture for the magnetic ground state of $\mathrm{Li}_{2} \mathrm{ZrCuO}_{4}$.

The well separated states around the Fermi energy are of main interest as they determine the magnetism of the system. These states are dominated by the in-plane orbitals of the $\mathrm{CuO}_{4}$ plaquettes corresponding to the antibonding $d p \sigma^{*}$ states (see two lower panels in Fig. 4). The distortion of the plaquettes from an ideal planar chain geometry does not affect significantly the band characters of the anti-bonding $d p \sigma^{*}$ states. The hybridization with out-of-plane states is very small and does not differ from compounds with ideal planar $\mathrm{CuO}_{2}$ chains.

In Fig. 5 the band structure of the valence band together with the $\mathrm{Cu} 3 d_{x^{2}-y^{2}}$ and $\mathrm{O} 2 p_{x, y}$ characters are shown. The anti-bonding $d p \sigma^{*}$ bands (see Fig. 6 left) show their main dispersion along the chain direction (Z$\Gamma)$ and in the chain plane $(\Gamma-Y, S-X)$ indicating the leading magnetic exchanges in the layers. Perpendicular to these directions the dispersion is small, suggesting small exchanges between the chain layers.

To unravel the hierarchy of exchanges in the system as well as to estimate a cut-off for negligible long range exchanges in further super-cell calculations, an effective one-band model was fitted to the anti-bonding $d p \sigma^{*}$ bands. Effective hoppings for $\mathrm{Cu}-\mathrm{Cu}$ distances up to $12 \AA$ are considered (see Fig. 6 right) and calculated using a least-square fit procedure. The resulting TB fit and the LDA bands are in perfect agreement (see Fig. 6 left).

All obtained transfer integrals larger than $5 \mathrm{meV}$ are summarized in Table [. We found the main hoppings between NN and NNN along the chains and three considerable inter-chain hoppings in the chain plane. Comparing the SYM results with results from the VCA model, we

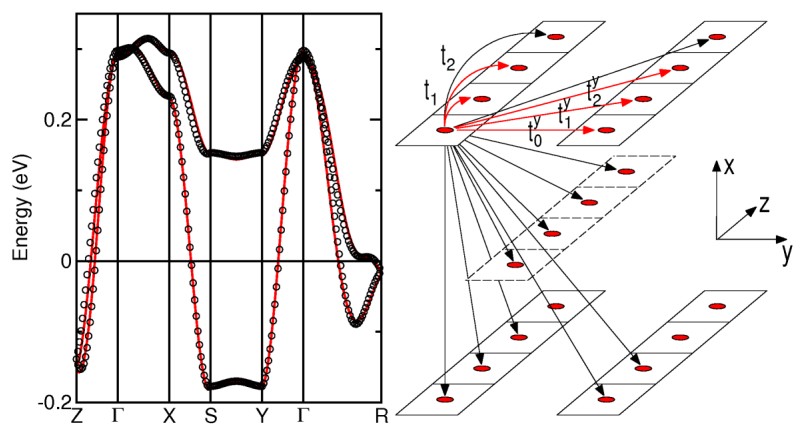

FIG. 6: (Color online) Left: Comparison of the calculated band structure (black dots) and the least-square fit (red line) of the TB model. Right: Considered hoppings for the TB model. The leading interactions are labeled and highlighted by red colors.

\begin{tabular}{llllll}
\hline \hline$t_{i} / \mathrm{meV}$ & $t_{1}$ & $t_{2}$ & $t_{0}^{y}$ & $t_{1}^{y}$ & $t_{2}^{y}$ \\
\hline SYM & -42 & -56 & -26 & 14 & -25 \\
VCA & -44 & -55 & -25 & 15 & -26 \\
FIC & -86 & -92 & -49 & 24 & -21 \\
\hline \hline
\end{tabular}

TABLE I: Resulting hopping integrals from TB fits for different structural models.

see that the differences in the hopping integrals are very small and in the same order as the accuracy of the TB fit procedure. This indicates that the chosen structural model for the $\mathrm{Li}(1)$ split position is irrelevant for the calculated coupling constants of $\mathrm{Li}_{2} \mathrm{CuZrO}_{4}$. Taking into account the strong Coulomb repulsion in a model approach we construct from our TB model a single-band Hubbard model (with a typical value $\underline{32} U_{\text {eff }}=4.2 \mathrm{eV}$ for edge shared $\mathrm{CuO}_{2}$ chains) which can be mapped subsequently onto a Heisenberg model. We find $J_{1}^{A F M}=1.7 \pm 0.1 \mathrm{meV}$ for the NN exchange and $J_{2}^{A F M}=3.0 \pm 0.1 \mathrm{meV}$ for the NNN exchange $\underline{33}$

The exchange parameters resulted from the effective TB model disregard the FM contributions to the exchanges which are, however, expected between NN plaquettes in the compound due to the close to $90^{\circ}$ bond angle $\mathrm{Cu}-\mathrm{O}-\mathrm{Cu}$ along the chains. Naturally, these FM interactions are included in $\mathrm{LDA}+U$ calculations. To evaluate $J_{1}$ and $J_{2}$ on a quantitative level, we performed a series of super-cell calculations. The comparison of the total energies of these super-cells with different spin arrangements 34 and a subsequent mapping onto a Heisenberg model leads to a FM NN exchange $J_{1}^{T O T}=-11.2$ $\mathrm{meV}$ and an AFM NNN exchange $J_{2}^{T O T}=2.2 \mathrm{meV}$. The ground states of the compound is determined by the ratio $\alpha=-J_{2}^{T O T} / J_{1}^{T O T}=0.22 \pm 0.03$ that would result in a FM ordered ground state in close vicinity of a QCP $(\alpha=0.25)$. The variation of $U_{3 d}$ from $5.5 \mathrm{eV}$ to $8.0 \mathrm{eV}$ in the $\mathrm{LDA}+U$ calculation does not change this behavior qualitatively, although for the smaller $U$ values a slightly increased $\alpha$ is obtained $\stackrel{35}{~ B e s i d e s ~ a ~}$ 


\begin{tabular}{lllllll}
\hline \hline$J_{i} / \mathrm{meV}$ & $J_{1}^{\text {TOT }}$ & $J_{2}^{\text {TOT }}$ & $J_{1}^{A F M}$ & $J_{1}^{F M}$ & $J_{2}^{A F M}$ & $J_{2}^{F M}$ \\
\hline SYM & -11.2 & 2.2 & 1.7 & -12.9 & 3.0 & -0.8 \\
VCA & -10.6 & 2.5 & 1.8 & -12.4 & 2.9 & -0.4 \\
FIC & -10.2 & 5.9 & 7.0 & -17.2 & 8.0 & -1.1 \\
\hline \hline
\end{tabular}

TABLE II: Total and partial exchange integrals for different structural models from $\mathrm{TB}$ model and $\mathrm{LDA}+U$ calculation $\left(U_{3 d}=7.5 \mathrm{eV}\right)$.

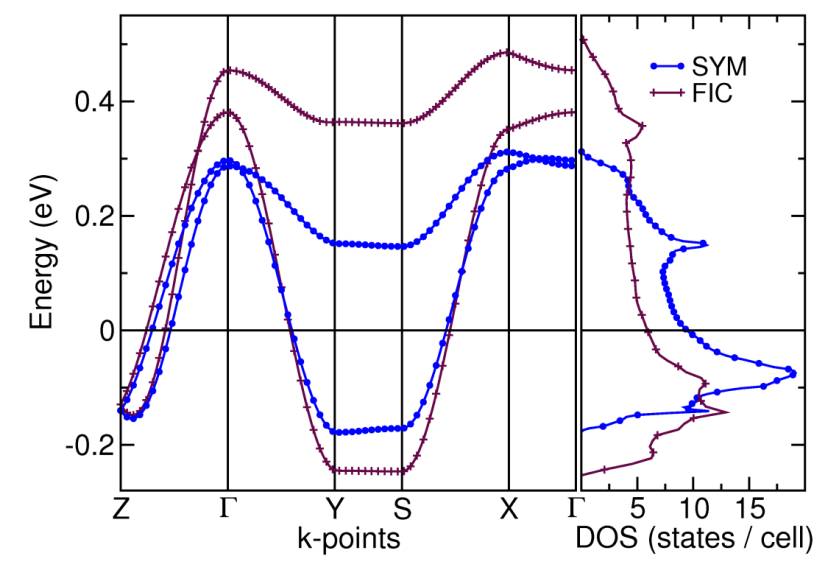

FIG. 7: (Color online) Comparison of band structure and density of states at Fermi energy for both chain geometries. The small displacement of $\mathrm{O}$ atoms from the mirror plane leads to a decrease of the band dispersion.

possible small error in the calculated exchange parameters, the experimentally observed spiral state from NMR data ${ }^{36}$ might result due to the sizable inter chain couplings $J_{0}^{y}=4\left(t_{0}^{y}\right)^{2} / U_{\text {eff }} \sim 0.6 \mathrm{meV}$ and $J_{2}^{y} \sim 0.6 \mathrm{meV}$ that both stabilize long range order.

\section{Influence of chain-buckling}

Whereas the different treatment of the $\operatorname{Li}(1)$ split position does not influence the band structure and the corresponding magnetic model essentially, the role of the distortion of the $\mathrm{CuO}_{4}$ plaquettes (see Fig. 1b) remains to be addressed: How much does the non-planar geometry of the chains influence the balance of the NN, NNN and inter-chain couplings and the resulting placement of the compound in the $J_{1}-J_{2}$ phase diagram, especially its vicinity to the QCP? For a fictitious planar chain, 30 changes are expected since a changed $\mathrm{Cu}-\mathrm{O}-\mathrm{Cu}$ bond angle (changed by about $0.8^{\circ}$ ) will influence $\mathrm{J}_{1}^{T O T}$ and the different orientation of the anti-bonding $p d \sigma^{*}$ "molecular orbital" will alter $J_{2}^{T O T}$. Since these changes are hardly predictable quantitatively from structural considerations only, we apply our mapping approach also to a fictitious compound with ideal planar $\mathrm{CuO}_{2}$ chains.

In Fig. 7 the resulting anti-bonding $d p \sigma^{*}$ bands and the related density of states for this fictitious structure are shown. For a planar chain geometry we obtain an about $50 \%$ larger bandwidth, but a very similar shape of the bands compared with the real structure. On first glance, this suggests a scaling of the leading hopping parameters, only. Our TB fit yields nearly a doubling of the hopping integrals as listed in Tab. II thus a dramatic change of the corresponding exchange terms can be expected. To take into account the large FM contributions to $J_{1}^{T O T}$, we carried out $\mathrm{LDA}+U$ calculations for the same supercells used before $\underline{\underline{34}}$

Surprisingly, we find a nearly unchanged NN exchange $\mathrm{J}_{1}^{T O T}$, but only due to the compensation of the increased individual contributions $J_{1}^{F M}$ and $J_{1}^{A F M}$ (see Tab. III). Since the FM contribution $J_{2}^{F M}$ to $J_{2}^{T O T}$ remained small - as for the real structure - it basically scales like $J_{2}^{A F M} \sim 4 t_{2} / U_{\text {eff }}$. Thus, we obtain a large change in $J_{2}^{T O T}$ and consequently in the frustration ratio $\alpha \sim 0.6$ for the planar chains. In consequence, the ratio $\alpha=-J_{2} / J_{1}$ depends strongly on structural details of the local $\mathrm{Cu}-\mathrm{O}$ environment. This is especially important regarding the vicinity of the system to the QCP. On the other hand this sensitivity of $\alpha$ to the chain buckling may provide the opportunity of manipulating the ground state of the system selectively by substitution or external pressure.

Unfortunately, a planar chain arrangement leads also to a sizable increase of the inter chain coupling. This can be understood as the decrease of buckling increases the inter-chain overlap of the $\mathrm{O}$ orbitals belonging to the $p d \sigma^{*}$ states. This way, a tendency towards long range order is stabilized, although the quantitative influence of inter-chain couplings to the ground state is sparsely considered in the literature.

\section{Stability of the $\operatorname{Li}(1)$ split position}

Although the different treatments of the $\mathrm{Li}(1)$ split position yield no significant influence on the magnetic exchange parameters the split position may influence other properties of the compound. Related to the split position, the local symmetry or disorder could modulate the pitch angle of the helical state or be crucial for the formation of a possible multiferroic phase. Furthermore, ${ }^{7} \mathrm{Li}$ NMR and complex dielectric measurements reveal a glass-like ordering of the $\operatorname{Li}(1)$ ions below $T_{g}=100 \mathrm{~K}{ }^{37}$ Thus, using our super-cells simulating different types of Li(1) order (see Fig. 2), we tackled the questions whether a tendency to a static $\operatorname{Li}(1)$ order can be supported by calculations.

For the purpose of computational feasibility, we restricted ourselves to a fourfold cell (32 atoms) $\stackrel{38}{=}$ The comparison of the total energy for the different ordered Li(1) patterns (SYM, S1, S2, S3) favors energetically the high-symmetry model (SYM) with a single $\mathrm{Li}(1)$ position. The second lowest in energy is the structure with alternating $\operatorname{Li}(1)$ displacement along the chain direction (S3). For this $\operatorname{Li}(1)$ arrangement, the dependence of the 


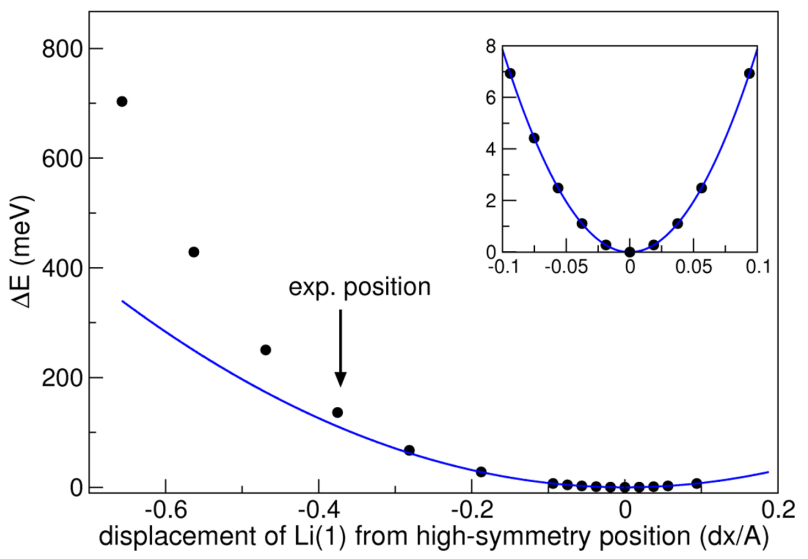

FIG. 8: (Color online) Energy difference depending on the displacement of the $\mathrm{Li}(1)$ atom from the high-symmetry position. The experimental observed displacement of $0.4 \mathrm{~A}$ is indicated by an arrow. The inset shows a harmonic (second order) fit to the calculated energy differences for small displacements from the equilibrium position $( \pm 0.1 \AA)$. The same fit is shown in the main panel, indicating deviations from the harmonic behavior at larger $\operatorname{Li}(1)$ elongations.

total energy on the displacement is depicted in Fig. 8 The resulting curve does not indicate a double well potential as expected for a $\mathrm{Li}(1)$ split position, but a minimum around the high symmetry position and a harmonic behavior (see inset Fig. 8) up to almost $0.3 \AA \mathrm{Li}(1)$ displacement. According to our calculations, for the experimentally suggested $\operatorname{Li}(1)$ split position (marked by the arrow in Fig. 8) only slight anharmonic effects could be expected. We also investigated whether the strong Coulomb repulsion on the $\mathrm{Cu}$ site could be the reason for the discrepancy between the single $\mathrm{Li}(1)$ position suggested by the calculations and the experimental split position using $\mathrm{LDA}+U$. With $\mathrm{U}_{3 d}$ in the range from 5 $7.5 \mathrm{eV}$ we do not obtain any significant changes of the position of the minimum and the slope of the curve, suggesting that the correlations at the $\mathrm{Cu}$ site play only a minor role for a possible $\operatorname{Li}(1)$ order.

So far our calculations with a fixed lattice (apart from Li(1)) can not support the split position, but favor the high-symmetry solution (SYM). The discrepancy with the experiment that observed a glass-like $\mathrm{Li}(1)$ ordering below $100 \mathrm{~K}$, may have the following origins: (i) dynamic effects that violate the adiabatic approximation used in the calculations or (ii) a correlated $\mathrm{Li}(1)-\mathrm{O}(1)$ position without long range order that would be difficult to detect in X-ray diffraction experiments. An investigation of these effects is beyond the scope of the present study but should be carried out in future since it might be crucial for the final picture of the compound.

\section{E. Cluster Calculations}

In order to provide an independent microscopic interpretation of the exchange integrals reported above, we performed exact diagonalization studies for the commonly used five-band extended $p d$ Hubbard model for finite open $\mathrm{Cu}_{n} \mathrm{O}_{2 n+2}$ clusters. The most reliable way to choose a reasonable parameter set by fitting various spectroscopic experiments cannot be used here due to the lack of single crystals and available experimental data. Since no parameter set for the five-band $p d$ model for a buckled chain geometry is available, we considered only the fictitious planar case (FIC) without any O-buckling. This way, a direct comparison to the band structure derived results (FIC) should be more reliable. Thus, we started from an available parameter set known for the well-studied closely related sister compound $\mathrm{Li}_{2} \mathrm{CuO}_{2}$

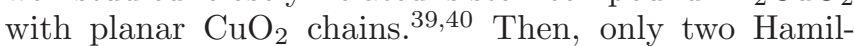
tonian parameters - the mean $\mathrm{Cu}-\mathrm{O}$ on-site difference $\Delta_{p d}=3.5 \mathrm{eV}(3.75 \mathrm{eV})$ and $K_{p d}=54 \mathrm{meV}(81 \mathrm{meV})$ - have to be slightly lowered to obtain consistency with the band structure results for the exchange integrals $J_{1}$ and $J_{2}$ reported above. For comparison the $\mathrm{Li}_{2} \mathrm{CuO}_{2}$ parameters are given in brackets 11 . The slightly smaller $\Delta_{p d}$ is in line with the slight increase of the $\mathrm{Cu}-\mathrm{O}$ distance by about $2 \%$ along the $\mathrm{CuO}_{2}$ chains for $\mathrm{Li}_{2} \mathrm{ZrCuO}_{4}$ compared to $\mathrm{Li}_{2} \mathrm{CuO}_{2}$.

With these parameters the title compound exhibits a charge gap of about $2.35 \mathrm{eV}$ as expected for an undoped cuprate. Therefore all eigenstates below that energy are spin states which can be described also reasonably well by a Heisenberg Hamiltonian. In case of a $\mathrm{Cu}_{5} \mathrm{O}_{10}$ cluster we deal with 10 spin states. Mapping for instance these 10 spin states onto a $1 \mathrm{D} J_{1}-J_{2}-J_{3}$-Heisenberg model ${ }^{42}$ we arrive then at $J_{1}=-10.6 \mathrm{meV}, J_{2}=5.9 \mathrm{meV}$ and $J_{3}=-0.3 \mathrm{meV}$ close to the values (FIC) given in Tab. II. Notice that our mapping on larger clusters with $5 \mathrm{Cu}-$ sites is considered to be more accurate than the determination of $J_{1}$ from a $\mathrm{Cu}_{2} \mathrm{O}_{4}$-dimer and $J_{2}$ from a $\mathrm{Cu}_{2} \mathrm{O}_{8}$ pseudo-trimer, where the central $\mathrm{Cu}$ has been removed, $\underline{43}$

In the context of the lower value of $J_{1}$ compared with Ref. 20 we would like to mention that from a microscopic point of view $J_{1}$ depends strongly on the adopted value of the direct ferromagnetic $\mathrm{Cu}-\mathrm{O}$ exchange parameter $K_{p d}$. Within perturbation theory it occurs already in the second order whereas the antiferromagnetic superexchange due to deviations from the $90^{\circ}$ and/or due to different crystal fields seen by the $\mathrm{O}$ orbitals along and perpendicular to the chain direction as well as the ferromagnetic Hund's-rule coupling and the O-sites occur in the fourth order, only 44 Analytically and numerically one obtains a linear dependence. Unfortunately, at present there is no consensus about a reliable value of $K_{p d}$ based on microscopic considerations. Therefore it is frequently treated as fitting parameter to reproduce empirical or otherwise determined values of $J_{1}$ just as done here. For other $\mathrm{CuO}_{2}$ edge-shared chain cuprates $K_{p d}$-values in between $50 \mathrm{meV}$ and $110 \mathrm{meV}$ have been 


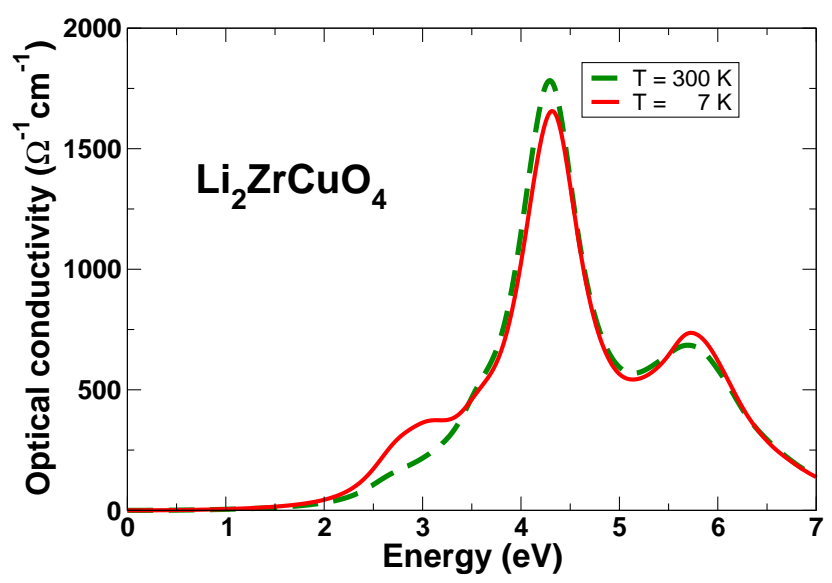

FIG. 9: (Color online) Optical conductivity of $\mathrm{Li}_{2} \mathrm{ZrCuO}_{4}$ within the five-band extended Hubbard model for a $\mathrm{Cu}_{5} \mathrm{O}_{12}$ cluster.

employed by various authors $20,39,40,43,45$ In our previous work on $\mathrm{Li}_{2} \mathrm{ZrCuO}_{4}{ }^{20} 70 \mathrm{meV}$ has been adopted, thus with the given above $54 \mathrm{meV}$ we are near to the lower bound of so far used $K_{p d}$-values. The former value was based on 1D-fits of the magnetic susceptibility $\chi(T)$ and of the magnetic specific heat $c_{v}(T) \approx c_{p}(T)$, which might be significantly affected by the ignored inter-chain interaction. Due to the vicinity to the critical point the calculated $1 \mathrm{D}$ saturation field at $T=0 \mathrm{~K}$ is very small of the order of 4 to 5 Tesla. Hence, the experimentally measured saturation field of about 30 Tesla (at low temperature) is clearly dominated by the inter-chain interaction in accord with our calculated sizable inter-chain couplings (see Sec. IVB).

In order to stimulate optical measurements and for a further experimental check of the applied Hamiltonian parameters we calculated (see Fig. 9) the in-chain optical conductivity for $300 \mathrm{~K}$ and $7 \mathrm{~K}$ using the technique presented in Ref. 39. We draw attention to the strong temperature effect near $3 \mathrm{eV}$ between low $(7 \mathrm{~K})$ and room temperature $(300 \mathrm{~K})$. This phenomenon is related to the different optical response in different initial spin states. For relatively small exchange integrals $J_{1}$ and $J_{2}$ the thermal population of these spin states changes markedly in the mentioned temperature range between zero and room temperature. Since the NN exchange $J_{1}$ (FIC) is mostly unchanged compared to the buckled structure (SYM, VCA), a strong temperature dependence of the optical conductivity should hold for the real compound.

Further investigations to settle the value of $J_{1}$ are highly desirable not only to get access to the Hamiltonian parameters,$\underline{46}$ but also to check for renormalization and creation of longer-ranged couplings due to strong enough spin-phonon interaction $\stackrel{47}{*}$ The strong coupling of the exchange parameter $J_{2}$ to the $\mathrm{O}$ buckling, indicated by our band structure calculations (see Tab.【I), opens room for such a scenario.

\section{CONCLUSIONS AND SUMMARY}

To summarize, applying LDA and LDA $+U$ band structure calculations as well as exact diagonalization studies of an extended $p d$ Hubbard model for large $\mathrm{Cu}_{n} \mathrm{O}_{2 n+2}$ clusters $(\mathrm{n}=5)$, that were finally all mapped onto a Heisenberg model, we obtain a consistent, microscopically based picture: (i) $\mathrm{Li}_{2} \mathrm{ZrCuO}_{4}$ can be understood as a quasi-1D chain compound with FM NN $J_{1}$ and AFM NNN $J_{2}$ exchange in close vicinity to a QCP. (ii) We find sizable inter-chain couplings that should be relevant for the magnetic ground state of the system, especially due to the vicinity of the system to a critical point where the impact of the leading exchange parameters on the ground state gets small. (iii) Calculations for a fictitious structure with planar chains indicate that the balance between $J_{1}$ and $J_{2}$ depends heavily on structural details, especially on the magnitude of the $\mathrm{CuO}_{2}$ chain buckling. (iv) This also applies for the inter-chain coupling which is strongly increased for the fictitious planar geometry.

Our findings suggest that the buckling of the $\mathrm{CuO}_{2}$ chain is crucial for the vicinity to the QCP, therefore it should be possible to tune the system towards that point by chemical substitution at the Zr site or by external pressure. On the other hand, it would be desirable to decrease the inter-chain coupling for which the orientation of the $\mathrm{CuO} p d \sigma$ orbitals is more relevant than interatomic distances according to our results. Thus, a search for good quasi 1D systems should turn towards crystal structures where the chains are arranged in a strongly non-planar pattern.

Our simulations indicate that the $\operatorname{Li}(1)$ split position has only a negligible direct influence on the spin system, assuming that all other atoms of the structure are fixed to their experimental position. In particular, our calculations do not support the experimental observation of a glassy-like order for $\mathrm{Li}(1)$ on this split position ${ }^{37}$ since it yields a single $\mathrm{Li}(1)$ position as most energetically favorable. However, an indirect influence of the $\operatorname{Li}(1)$ position on the spin system is possible: Taking into account a possible relaxation of the neighboring oxygen atom in the $\mathrm{CuO}_{2}$ chain could result in a sizable modification of the exchange parameters as shown by the strong influence of this $\mathrm{O}$ position on the leading exchange terms. Another possible origin of the discrepancy concerning the $\operatorname{Li}(1)$ position could arise from dynamic and non-adiabatic effects that are beyond the scope of the present study.

\section{ACKNOWLEDGMENT}

We acknowledge the DFG (Emmy-Noether program, H.R.) and the Grant DR269/3-1 (S.-L.D., J.M.), the German-Israel foundation (H.R.), and the ASCR project 
AVOZ10100520 (J.M.) for financial support. The ZIH and the IFW Dresden are acknowledged for the use of their computer facilities. We thank O. Janson, D. Kasi- nathan, H. Eschrig, N.M. Plakida, A.S. Moskvin, and R. Kuzian for fruitful discussions.
1 R. McKenzie, Sience 278, 820 (1997).

${ }^{2}$ G. Misguich and P. Sindzingre, European Physical journal B 59, 305 (2007).

3 D. Johnston, Normal-state magnetic properties of singlelayer cuprate high-temperature superconductors and related materials, vol. 10 (North-Holland, 1997).

${ }^{4}$ R. Melzi, P. Carretta, A. Lascialfari, M. Mambrini, M. Troyer, P. Millet, and F. Mila, Phys. Rev. Lett. 85, 1318 (2000).

${ }^{5}$ R. Melzi, S. Aldrovandi, F. Tedoldi, P. Carretta, P. Millet, and F. Mila, Phys. Rev. B 64, 024409 (2001).

${ }^{6}$ H. Rosner, R. Singh, W. Zheng, J. Oitmaa, S.-L. Drechsler, and W. Pickett, Phys. Rev Lett. 88, 186405 (2002).

7 H. Rosner, R. Singh, W. Zheng, J. Oitmaa, and W. Pickett, Phys. Rev. B 67, 014416 (2003).

8 A. Bombardi, J. Rodriguez-Carvajal, S. Matteo, F. de Bergevin, L. Paolasini, P. Carretta, P. Millet, and R. Caciuffo, Phys. Rev. Lett. 93, 027202 (2004).

9 R. Shpanchenko, V. Chernaya, A. A. Tsirlin, P. Chizhov, D. Sklovsky, E. Antipov, E. Khlybov, V. Pomjakushin, A. Balagurov, J. Medvedeva, et al., Chem. Mater. 16, 3267 (2004).

10 K. Oka, I. Yamada, M. Azuma, S. Takeshita, K. H. Satoh, A. Koda, R. Kadono, M. Takano, and Y. Shimakawa, Inorg. Chem. 47, 7355 (2008).

11 U. Nitzsche, S.-L. Drechsler and H. Rosner, to be published.

12 Recent inelastic neutron scattering data by W. Lorenz et al. (privat communication) yield $\alpha=0.31$ for $\mathrm{Li}_{2} \mathrm{CuO}_{2}$ and suggest that the ferromagnetic in-chain ordering in this compound is stabilized by the antiferromagnetic interchain interactions.

13 A. A. Gippius, E. N. Morozova, A. S. Moskvin, A. V. Zalessky, A. A. Bush, M. Baenitz, H. Rosner, and S.-L. Drechsler, Phys. Rev. B 70, 020406 (2004).

14 S.-L. Drechsler, J. Málek, J. Richter, A. S. Moskvin, A. A. Gippius, and H. Rosner, Phys. Rev. Lett. 94, 039705 (2005).

15 M. Enderle, C. Mukherjee, B. Fak, R. Kremer, J. Broto, H. Rosner, S.-L. Drechsler, J. Richter, J. Malek, A. Prokofiev, et al., Europhys. Lett. 70, 237 (2005).

16 T. Masuda, A. Zheludev, A. Bush, M. Markina, and A. Vasiliev, Phys. Rev. Lett. 92, 77201 (2004).

17 L. Capogna, M. Mayr, P. Horsch, M. Raichle, R. Kremer, M. Sonin, and B. Keimer, Phys. Rev. B 71, 140402 (2005).

18 S.-L. Drechsler, J. Richter, A. Gippius, A. Vasiliev, A. Bush, A. Moskvin, Y. Prots, W. Schnelle, and H. Rosner, Europhys. Lett. 73, 83 (2006).

19 M. Hase, I.Terasaki, and K. Uchinokura, Phys. Rev. Lett. 70, 3651 (1993).

20 S.-L. Drechsler, O. Volkova, A. N. Vasiliev, N. Tristan, J. Richter, M. Schmitt, R. Rosner, J. Málek, R. Klinger, A. A. Zvyagin, et al., Phys. Rev. Lett. 98, 077202 (2007).

21 C. Dussarrat, G. C. Mather, V. Caignaert, B. Domengès, J. G. Fletcher, and A. R. West, Journal of Solid State Chemistry 166, 311 (2002).
${ }^{22}$ The total energy calculations for the $\mathrm{Li}(1)$ position (Section IV D) have been carried out with both versions to ensure the independence from the basis set.

${ }^{23}$ K. Koepernik and H. Eschrig, Phys. Rev. B 59, 1743 (1999).

24 J. Perdew and Y. Wang, Phys. Rev. B 45, 13244 (1992).

25 J. Perdew, K. Burke, and M. Ernzerhof, Phys. Rev. Lett. 77, 3865 (1996).

26 H. Eschrig, K. Koepernik, and I. Chaplygin, J. Solid States Chemistry 176, 482 (2003).

27 Since in Ref. 21 and in the $\operatorname{ICSD}(59618)$ the lattice parameters and internal coordinates are inconsistent or erroneous, we explicitly provide the parameters used in the calculations: Space group Cccm (66), $a=9.385 \AA$, $b=5.895 \AA, c=5.863 \AA, \mathrm{Cu}(0.0,0.0,0.5), \operatorname{Li}(1)(0.21$, $0.75,0.0), \mathrm{Li}(2)(0.0,0.5,0.25), \mathrm{Zr}(0.25,0.25,0.0), \mathrm{O}(1)(-$ $0.0246,0.228,0.5), \mathrm{O}(2)(0.2662,0.0,0.25)$.

28 J. Kanamori, Theor. Phys. (Kyoto) 17, 177 (1957).

29 For the high-symmetry position (SYM) Li(1) is placed at $(0.25,0.75,0.0)$.

$30 \mathrm{O}(1)$ was placed at $(0.0,0.228,0.5)$. This structural variation decreases the $\mathrm{Cu}-\mathrm{O}$ bond length by $0.01 \AA$ and increases the $\mathrm{Cu}-\mathrm{O}-\mathrm{Cu}$ bond angle along the edge shared chain by $0.8^{\circ}$.

31 The experimentally determined distance between the two possible sites of a split position is $0.757 \AA$. For the VCA calculation the distance has been enlarged to $1.314 \AA$.

${ }^{32}$ F. Parmigiani, L. Sangaletti, A. Goldoni, U. del Pennino, C. Kim, Z.-X. Shen, A. Revcolevschi, and G. Dhalenne, Phys. Rev. B 55, 1459 (1996).

33 The error bars take into account the differences between the two Li(1) models (see Fig. 2 top) and the numerical errors of the fitting procedure.

${ }^{34}$ We doubled the unit cell along the chain direction and stabilized the following periodic spin arrangements to calculate $J_{1}$ and $J_{2}: \uparrow \uparrow \uparrow \uparrow, \uparrow \downarrow \uparrow \downarrow, \downarrow \uparrow \uparrow \uparrow$.

${ }^{35}$ For $U_{d}=5.5 \mathrm{eV}$ and $U_{d}=8.0 \mathrm{eV}$ we obtain $J_{1}=$ $-16.7 \mathrm{meV}, \quad J_{2}=4.1 \mathrm{meV}, \alpha=-0.25$ and $J_{1}=$ $-10.1 \mathrm{meV}, J_{2}=1.9 \mathrm{meV}, \alpha=-0.19$, respectively.

36 J. Kanamori, J. Phys. Soc. Jpn. 77, 043703 (2008).

37 E. Vavilova, A. Moskvin, Y. Arango, A. Sotnikov, V. Kataev, S.-L. Drechsler, O. Volkova, A. Vasiliev, and B. Büchner, arXiv: p. 0810.5754v2 (2008).

38 Besides the very small energy differences compared to the large total energy for different $\operatorname{Li}(1)$ elongations from the equilibrium position, the main problem for larger cells is the rather bad convergency behavior. The bad convergency is related to the well known problem of "charge shuffling" between (with respect to their charge density) almost identical, but crystallographically formaly different atoms.

39 J. Málek, S.-L. Drechsler, U. Nitzsche, H. Rosner, and H. Eschrig, Phys. Rev. B 78, 060508(R) (2008).

40 J. Málek, R. Kuzian, S. Nishimoto, M. Knupfer, and S.-L. Drechsler, (in preparation) (2009).

41 The remaining parameters of the five-band extended Hubbard model read $U_{d}=8.5 \mathrm{eV}, U_{p}=4.1 \mathrm{eV}, U_{p p}=$ 
$2.9 \mathrm{eV}, K_{p}=0.6 \mathrm{eV}$ (Hund's rule coupling on O-sites), $V_{p d}=0.65 \mathrm{eV}$ (inter-site Coulomb interaction, neglected in Ref. 39), $\varepsilon_{d}=0, \varepsilon_{p_{x}}=3.6 \mathrm{eV}$ (in-chain) and $\varepsilon_{p_{y}}=3.4 \mathrm{eV}$ (perpendicular to the chain). The transfer integrals read $t_{p_{y} d}=0.662 \mathrm{eV}, t_{p_{x} d}=0.765 \mathrm{eV}, t_{p_{x} p_{x}}=0.84 \mathrm{eV}$, $t_{p_{y} p_{y}}=-t_{p_{x} p_{x}} / 4$ in chain direction as well as $0.96 \mathrm{eV}$ and $-0.24 \mathrm{eV}$ in transversal direction (see also Ref. 43, notice the different notation of the chain axis (see Fig. 6).

42 The accuracy of the least-square fit for the differences between the Hubbard and Heisenberg eigen energies amounts $10^{-6}$.

43 Y. Mizuno, T. Tohyama, S. Maekawa, T. Osafune, N. Motoyama, H. Eisaki, and S. Uchida, Phys. Rev. B 57, 5326
(1998).

44 E. Eskes and A. Jefferson, Phys. Rev. B 48, 9788 (1993).

45 M. Braden, G. Wilkendorf, J. Lorenzana, M. Ain, G. McIntyre, M. Behruzi, G. Heger, G. Dhalenne, and A. Revolevschi, Phys. Rev. B 54, 1105 (1996).

46 Similar problems occur also for $J_{2}$ except the fact that it is hardly affected by $K_{p d}$ but by the much smaller value $K_{p p}$. In the present calculation we have therefore ignored $K_{p p}$ as it is usually done in the literature.

47 A. Weisse, G. Wellein, and H. Fehske, Phys. Rev. B 60, 6566 (1999). 